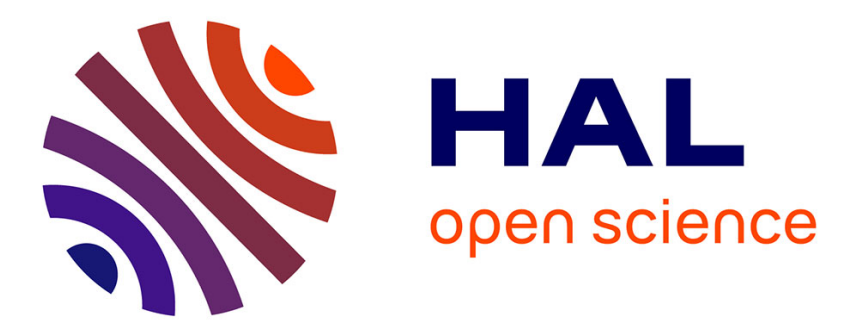

\title{
Spinal cord multi-parametric magnetic resonance imaging for survival prediction in amyotrophic lateral sclerosis
}

\author{
G. Querin, M. M. El Mendili, T. Lenglet, S. Delphine, V. Marchand-Pauvert, \\ H. Benali, Pierre-François Pradat
}

\section{To cite this version:}

G. Querin, M. M. El Mendili, T. Lenglet, S. Delphine, V. Marchand-Pauvert, et al.. Spinal cord multi-parametric magnetic resonance imaging for survival prediction in amyotrophic lateral sclerosis. European Journal of Neurology, 2017, 10.1111/ene.13329 . hal-01540346

\section{HAL Id: hal-01540346 https://hal.sorbonne-universite.fr/hal-01540346}

Submitted on 16 Jun 2017

HAL is a multi-disciplinary open access archive for the deposit and dissemination of scientific research documents, whether they are published or not. The documents may come from teaching and research institutions in France or abroad, or from public or private research centers.
L'archive ouverte pluridisciplinaire HAL, est destinée au dépôt et à la diffusion de documents scientifiques de niveau recherche, publiés ou non, émanant des établissements d'enseignement et de recherche français ou étrangers, des laboratoires publics ou privés. 


\title{
Spinal cord multi-parametric MRI for survival prediction in ALS
}

\author{
${ }^{1 *}$ Querin Giorgia, ${ }^{1 *}$ El Mendili Mohamed Mounir, ${ }^{2}$ Lenglet Timothée, S.Delphine, ${ }^{1}$ Marchand- \\ Pauvert Véronique, ${ }^{1}$ Benali Habib, ${ }^{1,3}$ Pradat Pierre-Francois
}

*First co-authors

${ }^{1}$ Sorbonne Universités, UPMC Univ Paris 06, CNRS, INSERM, Laboratoire d'Imagerie Biomédicale, Paris, France.

${ }^{2}$ APHP, Hôpital Pitié-Salpêtrière, Département de Neurophysiologie, Paris.

${ }^{3}$ APHP, Hôpital Pitié-Salpêtrière, Département des Maladies du Système Nerveux, Centre référent SLA, Paris, France.

\begin{abstract}
Background: Assessing survival is a critical issue in patients with Amyotrophic Lateral Sclerosis (ALS). Neuroimaging seems to be promising in the assessment of disease severity and several studies also suggest a strong relationship between spinal cord (SC) atrophy described by MRI and disease progression.

Aim of the study was than to determine the predictive added value of multimodal SC MRI on survival.

Methods: 49 ALS patients were recruited and clinical data collected. Patients were scored on ALSFRS-R and manual muscle testing. They were followed longitudinally to assess survival. Cervical spinal cord was imaged using 3T MRI system. Cord volume and cross-sectional area (CSA) at each vertebral level were computed. DTI metrics were measured. Imaging metrics and clinical variables were used as inputs for a multivariate Cox regression survival model.

Results: When building a multivariate Cox regression model with clinical and MRI parameters, FA, MTR, and CSA at C2-C3, C4-C5, C5-C6 and C6-C7 vertebral levels were significant. Moreover, hazard ratio (HR) calculated for CSA at C3-C4 and C5-C6 levels indicated an increased risk for patients with SC atrophy (respectively 0.66 and 0.68 ). In our cohort, MRI parameters seem to be more predictive than clinical variables, which had HR very close to 1 .

Conclusions: We suggest that multimodal SC MRI could be a useful tool in survival prediction especially if used at the beginning of the disease and when combined with clinical variables. To validate it as a biomarker, results confirmation in independent bigger cohorts of patients is warranted.
\end{abstract}

Total words count: 3587

Keywords: ALS, survival prediction, biomarkers, Spinal cord MRI, Spinal Cord Atrophy, motor neurons degeneration

The authors declare no conflict of interests. 


\section{Introduction}

Amyotrophic lateral sclerosis (ALS) is a fatal neurodegenerative disease involving both upper and lower motor neurons, leading to progressive limb paralysis and bulbar involvement. Mean survival time from disease onset is usually 3 to 5 years [1], with death occurring mostly secondary to respiratory failure. The disease is characterized by wide heterogeneity in the clinical presentation and in the progression rate [2], with some patients surviving 10 years or more $[3,4]$. For these reasons, there is an emerging need for specific biomarkers that could help in the characterization of clinical phenotypes and in prognosis evaluation.

Previous studies proposed different prognostic factors as determinant for survival estimation in ALS, including demographic and clinical parameters, the most reliable being age, site of onset, bulbar involvement, diagnostic delay, functional status and disability progression rate [5]. However, clinical and demographic variables do not allow reliable approximation in prognosis prediction when considered alone [6]. Combination of these variables with biochemical, neuroimaging and neurophysiological methods may increase the power of prediction. Within them, magnetic resonance imaging (MRI) techniques are used not only in the diagnostic process, but also as potential biomarkers of disease progression [7]. In comparison to brain imaging, spinal cord MRI has the advantage of investigating at the same time upper and lower motor neurons. However, spinal cord imaging has technical limitations due to the small diameter of the spinal cord, physiological motions (respiratory and cardiac movements) and susceptibility artefacts. In recent years, technical improvements, such as higher spatial resolution and respiratory/cardiac gating, allowed applying advanced MRI sequences to spinal cord studies [8]. Thanks to the improvement of spinal cord segmentation methods, it is now possible to accurately measure SC atrophy in many disease conditions, including motor neuron diseases [9]. By combining spinal cord MRI and electrophysiology (transcranial magnetic stimulation) in ALS patients, we showed that spinal cord atrophy was a marker of lower motor neuron degeneration, while diffusion tensor imaging (DTI) 
and magnetization transfer ratio (MTR) that measure white matter (WM) integrity were related to degeneration of corticospinal tracts (CST) [10]. Using multi-parametric MRI approaches combining DTI, MTR and atrophy measurements, several studies demonstrated abnormalities in the cervical cord of ALS patients compared to healthy controls [10-13]. Some of these abnormalities have been correlated with scores of clinical disability and with disease progression rate [14]; in particular, it has been demonstrated that atrophy rate is related with disease progression $[15]$ and that spinal cord $\underline{\text { cross-sectional area (CSA) and MTR are more reliable markers of longitudinal neurodegeneration }}$ compared to DTI metrics [8].

To further improve knowledge about the use of MRI parameters as early determinants of patients' prognosis and to determine if they could be predictive when used in combination with clinical parameters, we studied the possible correlation between MRI and survival in a cohort of 49 ALS patients.

\section{Methods}

\section{Subjects}

The local Ethics Committee of our institution approved all experimental procedures and written informed consent was obtained from each participant.

Forty-nine patients with probable or definite ALS were enrolled in the study and underwent a cervical cord MRI together with clinical and functional examination. Demographic data and clinical history of the patients were collected as well. The following variables were extracted for all patients from the center's database: gender; age at first symptoms; disease duration between symptoms onset and diagnosis; site of onset; body mass index (BMI) at first examination.

Revised ALS Functional Rating Scale (ALSFRS-R, 16) data were obtained. We calculated ALSFRS-R subscores pertaining to bulbar, upper limbs, truncal, lower limbs and respiratory function.

The progression rate at baseline of the ALSFRS-R score was defined by: 


$$
\Delta=\frac{A L S F R S_{\text {Max }}-A L S F R S_{M R I}}{\tau}
$$

With $\Delta$, the progression rate at baseline, $A L S F R S_{\text {Max }}=48$, the ALSFRS-R score for a healthy subject, $A L S F R S_{M R I}$, the ALSFRS-R score at MRI, and $\tau$, the delay between first symptoms and MRI (in months).

The patients were also scored on manual muscle testing (MMT) using the Medical Research Council score [17]. Seven muscles in each limb were assessed and a total score for upper and lower limbs was calculated.

Patients were followed for mortality both through contacts with their families or treating physicians and through the national mortality register until May 312016 . Mortality was taken as the time of death or of tracheostomy.

\section{MRI acquisition and data processing}

Acquisitions were performed using a 3T MRI system (TIM Trio, Siemens Healthcare, Erlangen, Germany). Anatomical imaging was performed at the cervical and upper thoracic spinal cord levels. DTI and magnetization transfer imaging were performed at the cervical levels from C2 to $\mathrm{C} 7$ using the following protocol:

- Anatomical data were acquired using a sagittal T2-weighted three-dimensional turbo spin echo (TSE) image with slab selective excitation. Imaging parameters were: isotropic voxel size $0.9 \times 0.9 \times 0.9 \mathrm{~mm}^{3} ; \mathrm{FOV}=280 \times 280 \mathrm{~mm}^{2} ; 52$ sagittal slices; TR $=1500 \mathrm{~ms} ; \mathrm{TE}=120 \mathrm{~ms}$; acceleration factor $=3$; acquisition time $=6 \mathrm{~min}$.

CSA was measured by an operator experienced on the segmentation technique on the T2-TSE images in the middle of vertebral levels. Data were segmented using a double threshold-based method (DTbM), an improved version of the well-established threshold-based method (TbM), with minimal manual intervention $[18,19]$. This technique has been previously validated by our group in 
healthy subjects and in patients affected by various neurological diseases including motor neuron diseases [18]. It has been demonstrated to be highly accurate at the cervical cord region [19].

- DTI [20] data were acquired using a single shot EPI sequence with monopolar diffusion weighting scheme to achieve low TE. The acquisition was cardiac-gated. Imaging parameters were: voxel size $=1 \times 1 \times 5 \mathrm{~mm}^{3} ; \mathrm{FOV}=128 \times 128 \mathrm{~mm}^{2} ; \mathrm{TR}=700 \mathrm{~ms} ; \mathrm{TE}=96 \mathrm{~ms} ;$ acceleration factor $=2 ;$ bvalue $=$ $1000 \mathrm{~s} / \mathrm{mm}^{2} ; 64$ diffusion encoding directions; 4 averages; acquisition time $=15 \mathrm{~min}$.

Data were corrected for motion slice-by-slice using FSL FLIRT [20] with three degrees of freedom (Tx, Ty, Rz). Diffusion metrics were estimated voxel-wise using FSL DTIFIT: fractional anisotropy (FA), radial diffusivity $(\lambda \perp)$, axial diffusivity $\left(\lambda_{/}\right)$and mean diffusivity (MD).

- 3D gradient echo images with slab-selective excitation were acquired with and without MT saturation pulse (Gaussian envelope, duration $=9984 \mu \mathrm{s}$, frequency offset $=1200 \mathrm{~Hz}$ ). $\underline{\text { Imaging }}$ parameters were: voxel size $=0.9 \times 0.9 \times 2 \mathrm{~mm}^{3} ; \mathrm{FOV}=230 \times 230 \mathrm{~mm}^{2} ;$ axial orientation with 52 slices (covering the same $\mathrm{C} 2$-T2 region as the DTI scans), flip angle $=23^{\circ}, \mathrm{TR}=28 \mathrm{~ms} ; \mathrm{TE}=3.2 \mathrm{~ms}$; acquisition time, 5 min per volume. MTR was computed voxel-wise following the equation MTR = $100 \times\left(\mathrm{S}_{0}-\mathrm{S}_{\mathrm{MT}}\right) / \mathrm{S}_{0}$, where $\mathrm{S}_{0}$ and $\mathrm{S}_{\mathrm{MT}}$ represent the signal without and with the magnetization transfer pulse, respectively.

The lateral portion of the cord, that includes mostly the CST, was delineated manually and using geometry-based information by an experienced operator on segmentation. To minimize bias, these regions of interest (ROIs) were defined on the mean diffusion weighted images (for DTI analysis) and on the 3D gradient-echo T1-weighted image (for MT analysis).

Figure 1 describes an example of multi-parametric cervical SC MRI in an ALS patient. We show both $\mathrm{T} 1$ and $\mathrm{T} 2$ weighted images representative of those used for atrophy measurements and estimation of DTI and MTR data.

\section{Statistical analysis}


Statistical analysis was performed using JMP 12. Demographic and clinical data were described as mean \pm standard deviation (SD). FDR (false discovery rate) correction was used for multiple correlations.

For survival analyses, participants were followed from entry to death, date of censoring alive or May 31 2016, whichever occurred first. Survival was plotted by baseline characteristics using the Kaplan-Meier method. Cox proportional hazards models were used to estimate hazard ratios (HRs) and $95 \%$ confidence intervals (CIs).

We first conducted univariate analyses in which each covariate was entered separately. We then performed multivariate analyses including MRI parameters and significant variables associated with mortality. We used a backwards stepwise selection procedure with a p-value of 0.10 for staying in the model. The significance level was set at $\mathrm{p}<0.05$.

\section{Results}

Demographic and clinical data of the patients are summarized in Table 1 while descriptive statistic relative to MRI parameters is summarized in Table 2.

\section{Survival analysis}

Of the 49 patients, 30 had died by the end of the study (61\%). Median survival time from MRI to death was $40.19 \pm 22.44$ months. Median survival time was 42.53 months. Age at MRI was $55.52 \pm 9.80$ years. No significant difference in survival was found when dividing the patients after the mean age at MRI.

General survival of the observed population was described using Kaplan-Meyer method as shown in Figure 1. 
We performed a univariate analysis using a Cox proportional hazard models to find out which clinic-demographical variables influenced significantly survival. Total MMT $(p=0.005)$ and MMT for upper limbs $(\mathrm{p}=0.025)$, progression rate at baseline of the ALSFRS-R score $(\mathrm{p}=0.04)$ and disease duration between symptoms onset and diagnosis $(\mathrm{p}=0.007)$ resulted to influence significantly survival.

\section{Multivariable Cox Proportional Hazards Models}

We fit a multivariate Cox regression model using clinical variables, which resulted significant in the univariate analysis together with the MRI variables. A significance level of 0.10 was used as cut off to stay in the model.

Total MMT at MRI and rate of ALSFRS-R declining were predictors of survival in the final model. Within MRI parameters FA $(\mathrm{p}=0.008)$, MTR $(\mathrm{p}=0.01)$, and CSA at C2-C3, C4-C5, C5-C6 and C6-C7 vertebral levels (respectively $\mathrm{p}=0.03, \mathrm{p}=0.03, \mathrm{p}=0.01, \mathrm{p}=0.02$ ) were significant. The hazard ratio (HR) calculated for CSA at C3-C4 and C5-C6 levels indicated an increased risk for patients with spinal cord atrophy at these levels (respectively $\mathrm{HR}=0.66, \mathrm{p}<0.001, \mathrm{CI}=[0.43$ 1.00]; and $\mathrm{HR}=0.68, \mathrm{p}<0.001, \mathrm{CI}=\left[\begin{array}{ll}0.46 & 0.90]\end{array}\right]$. They were more predictive than clinical variables which had $\mathrm{HR}$ very close to 1 , indicating a poor prediction power: $\mathrm{HR}=0.94$ for disease duration from onset to MRI (CI 0.86-1.1), 1.1 for ALSFRS-R progression rate $\left(\mathrm{CI}=\left[\begin{array}{ll}1.03 & 1.23\end{array}\right]\right)$ and 0.94 for total MMT $(\mathrm{CI}=[0.900 .97])$.

\section{Discussion}

Our study suggests that multi-parametric cervical spinal cord MRI is useful for survival prediction in ALS patients. Indeed, we describe MRI parameters as predictive of survival in our cohort, with HR related to CSA indicating a relation between focal cervical cord atrophy and shorter disease duration. 
From a pathological point of view, ALS is characterized by simultaneous degeneration of both upper and lower motor neurons and the complex clinical picture of the disease is determined by combination of the two of them [21, 22]. Description of changes over time both in brain and spinal cord has been proposed and, in recent years, neuroimaging has come out as a valid noninvasive tool in the analysis of pathological disease evolution. Degeneration in spinal cord manifests as involvement of both white matter of CST and grey matter (GM) related to the loss of motor neurons [23]. These changes alter the diffusivity properties of the cord, usually manifesting as progressive increase in MD and decrease in FA [8]. In a recent study, it has been shown that abnormalities in cervical spinal cord detected combining DTI, MTR, and atrophy measurements correlated with functional impairment [14].

Starting from these previous observations, we aimed at demonstrating that MRI parameters can be supportive also in prognosis evaluation. We show that CSA reduction in the cervical cord is associated with shorter survival. Respiratory dysfunction, which is the main cause of death in ALS patients, is closely related to the degeneration of motor neurons innervating diaphragm that are located in the cervical cord. At the same time, we demonstrate that FA and MTR values also influence survival, in accordance with the role of white matter involvement in the complex $\underline{\text { neurological picture and progression rate in ALS. Here we propose that MRI parameters referring to }}$ $\underline{\text { WM and GM degeneration should be considered together since they mirror the complexity of }}$ pathological changes happening in vivo. Such complexity and the fact that degeneration involves many systems at the same time account for the wide variability in clinical phenotypes and for the difficulty in building accurate models predicting survival. In fact, even though research in ALS has been progressing in many fields; a reliable biomarker and predictor of prognosis is still missing [24]. Nevertheless, it would be of primary importance in better management of patients and in patients' stratification during clinical trials [25]. Spinal cord MRI could be an effective biomarker and would be widely disposable and non-invasive. 
One limitation of our study is that patients with great disability or important bulbar or respiratory involvement are often not able to lie down for a long time within the MRI scanner. Due to this classical selection bias, common to all neuroimaging studies in ALS patients, our population may not be completely representative of a whole ALS population which limits the generalization of the results. Another limitation is that we did not include brain MRI, which has been recently shown to predict survival using deep learning [26]. Combination with brain MRI as well as more complex models such as deep learning could improve prediction and should be explored in future works.

In conclusion, our data support the hypothesis that spinal cord MRI could be useful when performed in patients at the beginning of the disease to better classify them and to recognize slow progressing from fast progressing patients. However, the validation of a biomarker is a complex and multistep process [27], which requires the confirmation of the results in big and independent populations. For this reason, our data will need further validation in independent and larger cohorts of patients.

Funding: This study was supported by the Association Française contre les Myopathies-Téléthon (AFM-Téléthon) and the Institut pour la Recherche sur la Moelle épinière et l'Encéphale (IRME). The research leading to these results has also received funding from the program “Investissements d'Avenir" ANR-10-IAIHU-06.

Acknowledgments: We thank the neurologists of the Paris ALS center who participated in the recruitment of the patients in the study: Drs Nadine Le Forestier, Vincent Meininger, Gaelle Bruneteau; François Salachas, Maria del Mar Amador and Rabab Debs. We thank Sophie Blancho 
for her help in the management and coordination of the study. We deeply acknowledge the patients who participated in this research.

\section{References}

1) Robberecht $\mathrm{W}$ and Philips $\mathrm{T}$. The changing scene of amyotrophic lateral sclerosis. Nat Rev Neurosci 2013; 14: 248-264. doi: 10.1038/nrn3430.

2) Swinnen B and Robberecht W. The phenotypic variability of amyotrophic lateral sclerosis. Nat Rev Neurology 2014; 10: 661-670. doi: 10.1038/nrneurol.2014.184.

3) Paganoni S, Macklin EA, Lee A, Murphy A, Chang J, Zipf A et al. Diagnostic timelines and delays in diagnosing amyotrophic lateral sclerosis. Amyotroph Lateral Scler Frontotemporal Deg 2014; 15: 453-456. doi: 10.3109/21678421.2014.903974.

4) Labra J, Menon P, Byth K, Morrison S, Vucic S. Rate of disease progression: a prognostic biomarker in ALS. J Neurol Neurosurg Pychiatry 2016; 87: 628-632. doi: 10.1136/jnnp2015-310998.

5) Gordon PH, Salachas F, Lacomblez L, LeForestier N, Pradat PF, Bruneteau G, et al. Predicting Survival of Patients with Amyotrophic Lateral Sclerosis at Presentation: A 15Year Experience. Neurodegenerative Dis 2013; 12:81-90. doi: 10.1159/000341316.

6) Chiò A, Logroscino G, Hardiman O, Swingler R, Mitchell D, Beghi E, et al. Prognostic factors in ALS: a critical review. Amyiotroph Laterat Scler 2009, 10: 310-232. doi: $10.3109 / 17482960802566824$.

7) Foerster BR, Welsh RC, Feldman EL. 25 years of neuroimaging in amyotrophic lateral sclerosis. Nat Rev Neurol. 2013; 9(9): 513-524. doi:10.1038/nrneurol.2013.153. 
8) Agosta F, Rocca MA, Valsasina P, Sala S, Caputo D, Perini M, et al. A longitudinal diffusion tensor MRI study of the cervical cord and brain in amyotrophic lateral sclerosis patients. J Neurol Neurosurg Psychiatry 2009; 80:53-55. doi: 10.1136/jnnp.2008.154252.

9) El Mendili MM, Chen R, Tiret B, Villard N, Trunet S, Pélégrini-Issac M, et al. Fast and accurate semi-automated segmentation method of spinal cord MR images at 3T applied to the construction of a cervical spinal cord template. PLoS One. 2014; 22;9(4): e95516. doi: 10.1371/journal.pone.0095516

10) Cohen-Adad J, Mendili MM, Morizot-Koutlidis R, Lehéricy S, Meininger V, Blancho S, et al. Involvement of spinal sensory pathway in ALS and specificity of cord atrophy to lower motor neuron degeneration. Amyotroph Lateral Scler Frontotemporal Degener 2013; 14:3038. doi: 10.3109/17482968.2012.701308.

11) Cohen-Adad J, Zhao W, Keil B, Ratai EM, Triantafyllou C, Lawson R, et al. 7-T MRI of the spinal cord can detect lateral corticospinal tract abnormality in amyotrophic lateral sclerosis. Muscle Nerve 2013;47:760-2. doi: 10.1002/mus.23720.

12) Nair G, Carew JD, Usher S, Lu D, Hu XP, Benatar. Diffusion tensor imaging reveals regional differences in the cervical spinal cord in amyotrophic lateral sclerosis. Neuroimage 2010; 53: 576-583. doi: 10.1016/j.neuroimage.2010.06.060.

13) Valsasina P, Agosta F, Benedetti B, Caputo D, Perini M, Salvi F, et al. Diffusion anisotropy of the cervical cord is strictly associated with disability in amyotrophic lateral sclerosis. $\mathrm{J}$ Neurol Neurosurg Psychiatry 2007; 78: 480-484. doi: 10.1136/jnnp.2008.154252.

14) Branco LM, De Albuquerque M, De Andrade HM, Bergo FP, Nucci A, França MC Jr. Spinal cord atrophy correlates with disease duration and severity in amyotrophic lateral sclerosis. Amyotroph Lateral Scler Frontotemporal Degener. 2014; 5(1-2):93-97. doi: 10.3109/21678421.2013.852589.

15) El Mendili MM, Cohen-Adad J, Pellegrini-Issac M, Rossignol S, Morizot-Koutidis R, Marchand-Pauvert V, et al. Multi-Parametric Spinal Cord MRI as Potential Progression 
Marker in Amyotrophic Lateral Sclerosis. PLoS One 2014; 22; 9 (4): e95516. doi: 10.1371/journal.pone.0095516.

16) Cedarbaum JM, Stambler N, Malta E, Fuller C, Hilt D, Thurmond B, Nakanishi A. The ALSFRS-R: a revised ALS functional rating scale that incorporates assessments of respiratory function. BDNF ALS Study Group (Phase III). J Neurol Sci 1994; 169: 13-21.

17) Great Lakes ALS Study Group. A comparison of muscle strength testing techniques in amyotrophic lateral sclerosis. Neurology 2003; 61: 1503-1507.

18) El Mendili M, Chen R, Tiret B, Pélégrini-Issac M, Cohen-Adad J, Lehéricy S, et al. Validation of a semiautomated spinal cord segmentation method. J Magn Reson Imaging. 2015 Feb;41(2):454-9. doi: 10.1002/jmri.24571.

19) El Mendili M, Lenglet T, Stojkovic T, Behin A, Guimares-Costa R, Salachas F, et al. Cervical spinal cord atrophy profile in adult SMN1-linked SMA. Plos One 2016; 11(4): e0152439. Doi: 10.137/journal.pone.0152439.

20)Cohen-Adad J, El Mendili MM, Lehéricy S, Pradat PF, Blancho S, Rossignol S, et al. Demyelination and degeneration in the injured human spinal cord detected with diffusion and magnetization transfer MRI. Neuroimage 2011; 55: 1024-1033. doi: 10.1016/j.neuroimage.2010.11.089.

21) Lawyer T, Netsky MG. Amyotrophic lateral sclerosis. A clinicoanatomic study of 53 cases. Arch Neurol Psychiatry 1953; 69:171-92.

22) Cruz-Sanchez FF, Moral A, Tolosa E, de Belleroche J, Rossi ML. Evaluation of neuronal loss, astrocytosis and abnormalities of cytoskeletal components of large motor neurons in the human anterior horn in aging. J Neural Transm 1998; 105:689-701.

23) Verstraete E, Polders DL, Mandl RC, Van der Heuvel MP, Veldink JH, Luijten P, et al. Multimodal tract-based analysis in ALS patients at 7T: a specific white matter profile? Amyotroph Lateral Scler Frontotemporal Degener 2014;15: 84-92. doi: $10.3109 / 21678421.2013 .844168$ 
24) Blasco H, Vourc'h P, Pradat PF, Gordon PH, Andres CR, Corcia P. Further development of biomarkers in amyotrophic lateral sclerosis. Expert Rev Mol Diagn. 2016; 16(8):853-68. doi: 10.1080/14737159.2016.1199277.

25) Menke RAL, Agosta F, Grosskreutz J, Filippi M, Turner MR. Neuroimaging Endpoints in Amyotrophic Lateral Sclerosis. Neurotherapeutics 2016; doi:10.1007/s 13311-016-0484-9.

26) Van der Burgh HK, Schmidt R, Westeneng HJ, de Reus MA, van den Berg LH, van den Heuvel MP. Deep learning predictions of survival based on MRI in amyotrophic lateral sclerosis. Neuroimage Clin. 2016; 13:361-369. doi: 10.1016/j.nicl.2016.10.008.

27) Grolez G, Moreau C, Danel-Brunaud V, Delmaire C, Lopes R, Pradat PF, et al. The value of magnetic resonance imaging as a biomarker for amyotrophic lateral sclerosis: a systematic review. BMC Neurol. 2016; 16(1):155. doi: 10.1186/s12883-016-0672-6. 